\title{
APROXIMACIÓN A LA LENGUA DE SEÑAS COSTARRICENSE (LESCO)
}

Priscilla Retana

\begin{abstract}
RESUMEN
En este trabajo, se presenta una reseña histórica de la Lengua de Señas Costarricense (LESCO). Además, se muestran los alcances que se realizaron en las investigaciones iniciales de esta lengua, con el fin despertar el interés por ella y desmitificar ciertas creencias populares, que aún en la actualidad la acompañan.; por ejemplo, el mito de la universalidad y la iconicidad y transparencia, Palabras clave: lenguas de señas, LESCO, reseña histórica, mitos, investigaciones sobre la lengua de señas.
\end{abstract}

\begin{abstract}
This article presents a historical overview of Costa Rican Sign Language (Lengua de Señas Costarricense: LESCO). It also reviews the impact of the early research of this language with the goal to stimulate interest in it and demystify certain popular beliefs that are still currently held; for example, the myths of universality, iconicity, and transparency.
\end{abstract}

Key words: sign language, LESCO, historical overview, myths, research on sign language.

\section{Introducción}

Para abordar el estudio de una lengua que aún no había sido descrita en la década de 1990 en Costa Rica, fue necesario revisar la literatura existente en otras lenguas de señas, especialmente en la lengua de señas norteamericana (LSN). Gracias al apoyo de la Dra. Soledad Chavarría, quien fungía como Directora del Programa Regional de la Sordera (PROGRESO) realicé una pasantía a la Universidad de Gallaudet en Washington para consultar las fuentes bibliográficas que allí existían ${ }^{1}$.

M.L. Priscilla Retana. Profesora retirada.

Correo electrónico: pretana1@gmail.com

Recepción: 12- 03- 2012

Aceptación: 20- 04- 2012 
Por otra parte, esta investigación no la hubiera podido llevar a cabo sin la ayuda de muchos de los miembros de la comunidad sorda. Precisamente, el día de hoy recuerdo con mucha gratitud a Allan Fernández Quesada, quien fue un líder muy querido y respetado por sus aportes y difusión de la cultura sorda. Allan no sólo me abrió las puertas de la comunidad sorda, sino que fue mi profesor de LESCO y como informante me apoyó desinteresadamente en el transcurso de mi investigación.

Deseo aprovechar este encuentro para compartir con ustedes los alcances que se realizaron en las investigaciones iniciales de esta lengua, con el fin despertar el interés por ella y desmitificar ciertas creencias populares: que aún en la actualidad la acompañan.

\section{Algunos mitos relacionados con las lenguas de señas}

Sobre este tipo de lenguas se han generado concepciones erróneas sustentadas en general por el escaso conocimiento o por prejuicios. A continuación, se hará referencia a dos de estos mitos.

\subsection{Iconicidad y transparencia}

Cuando un individuo se enfrenta por primera vez con una lengua visogestual ${ }^{2}$, percibe $^{2}$ que algunas señas como COMER, DORMIR, CASA, VACA, ÁRBOL, BEBER tienen un significado muy transparente y posiblemente considere que estas no son signos arbitrarios como las palabras sino que existe un alto grado de semejanza entre la forma física de la seña y el objeto que representa.

En el caso de la seña CASA, los dedos de ambas manos se encuentran extendidos y juntos, el pulgar también está en contacto con el resto de la mano. Con esta configuración manual, la señante realiza un movimiento simétrico frente a su cuerpo, y ambas manos se unen en las yemas de varios de sus dedos. La realización de esta seña aparentemente representa la forma de un techo de dos aguas. Por otra parte, en la seña VACA, la forma de la mano 'L' podría asociarse con el cuerno de este animal (Figura 1) ${ }^{3}$.

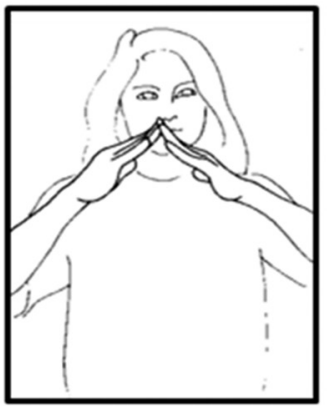

CASA

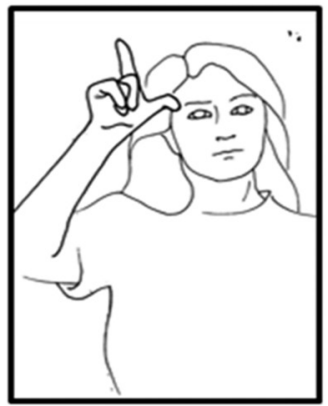

VACA

Figura 1. Iconicidad y transparencia en las lenguas de señas

Obsérvese otro ejemplo, en este caso propuesto por Klima y Bellugi (Cfr. 1979: 21) cuando estudian el grado de iconicidad en las lenguas de señas. En esta imagen, se observan tres señas para referirse a ÁRBOL (Figura 2): 


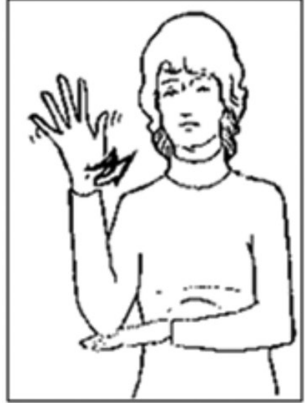

Lengua de señas norteamericana

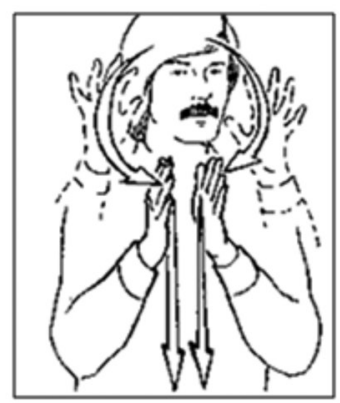

Lengua de señas danesa

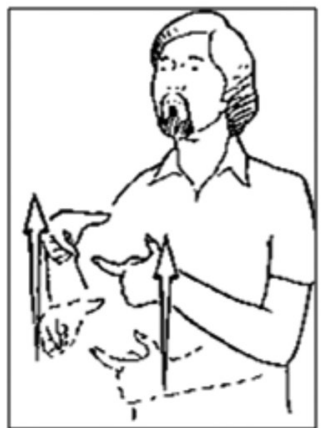

Lengua de señas china

Figura 2. Representación de la forma léxica 'ÁRBOL' en tres lenguas distintas

a. En la lengua de señas norteamericana, esta seña se realiza apoyando sobre el dorso de la mano pasiva el codo de la mano activa, la mano se mantiene abierta mientras gira. Se podría pensar que el antebrazo representa al tronco y el movimiento de la mano abierta representa el follaje moviéndose con el viento.

b. En la lengua de señas danesa, la seña difiere en todos los detalles de la ilustración anterior, pero también es icónica: las dos manos trazan simétricamente la forma de las ramas y el tronco.

c. Y en la lengua de señas china se observa una configuración manual diferente, pero la seña también es icónica: las dos manos abarcan la forma del tronco del árbol y realizan un movimiento ascendente.

En todos los casos expuestos, las señas aparentemente tienen un alto grado de iconicidad con el objeto representado, pero estas son arbitrarias y convencionales. De acuerdo con Stokoe 4 (Cfr. Retana 1993), estas se constituyen con base en determinadas propiedades o parámetros, como son la forma de la mano, su localización con respecto al cuerpo del señante y su movimiento. Estos componentes se realizan de forma simultánea, tienen un carácter fonológico y arrojan información sobre la estructura de las señas. Al igual que en una lengua oral como el español, se encontraron pares mínimos en la variedad de la lengua de señas usada en San José en 1990. Es muy posible que algunas de estas señas presenten alguna variación en la actualidad.

Obsérvese el par mínimo VACA: VENADO, en el cual las señas se oponen solo en la configuración manual (Figura 3):

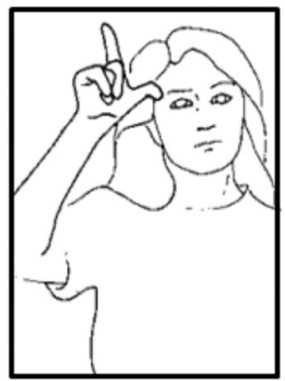

VACA

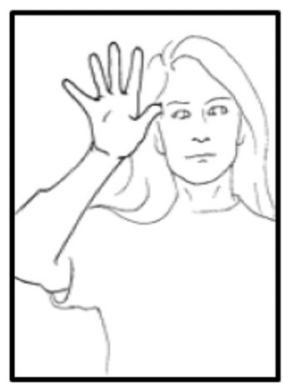

VENADO

Figura 3. Señas que solo contrastan en la configuración manual 
Al respecto, lingüistas como Battison (1980), Klima y Bellugi y Pietrosemoli (1988), entre otros, consideran que la iconicidad no juega un papel importante en la estructura y uso de la lengua. Esta posición se apoya en estudios como el realizado por Battison y Jordan (1980), en el cual se indica lo siguiente:

a. Si fueran icónicas, cualquier oyente entendería fácilmente las señas con sólo recibir una breve instrucción. Sin embargo, quienes están en el proceso de aprendizaje de una lengua de señas pueden constatar que se necesita mucho tiempo, esfuerzo y motivación para llegar a ser tan fluidos como en su lengua de nativa.

b. Las señas parecen icónicas cuando se realizan fuera de un contexto determinado, pero para un no-señante le es imposible entenderlas cuando forman parte de una conversación; primero, por la velocidad con que se realizan y segundo, porque la forma básica de las señas se altera al realizarse en una oración por la aplicación de procesos morfológicos y sintácticos.

Para ilustrar, veamos cómo se oscurece la iconicidad de las señas CASA y ANIMAL al formar el compuesto CASA ANIMAL cuya glosa equivale a 'granja' (Figura 4):

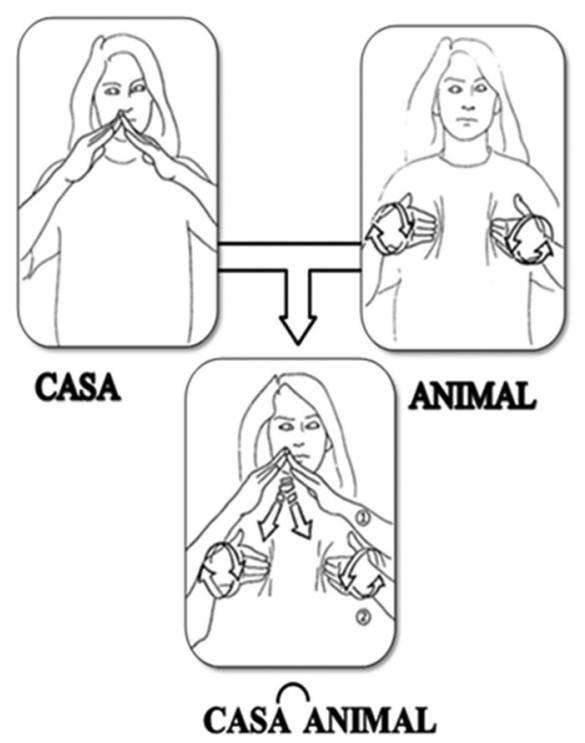

Figura 4. Formación de la seña 'GRANJA'

La formación del compuesto se manifiesta al acortar el espacio físico entre ambas señas y aumentar la velocidad entre el linde final de la primera y el linde inicial de la segunda. Además, el significado varía.

Por otra parte, el compuesto funciona como un todo, por lo tanto, se le puede anteponer o posponer un modificador para expresar la pluralidad, por ejemplo, tal y como se ilustra en los siguientes casos:

- JUAN CONSTRUIR CASA ANIMAL

'Juan construye la granja'

- JUAN CONSTRUIR MUCHO CASA ANIMAL

'Juan construye muchas granjas' 


\section{- JUAN CONSTRUIR CASA ANIMAL MUCHO}

'Juan construye muchas granjas'

Pero, si el modificador se coloca entre los constituyentes del compuesto, estos se comportan como unidades independientes y el modificador ya no califica al compuesto, como si fuera una sola unidad, sino a la forma nominal que le sigue.

- JUAN CONSTRUIR CASA MUCHO ANIMAL

'Juan construye la casa para muchos animales'

\subsection{Universalidad}

La creencia de que la lengua de señas es la misma para todo el mundo todavía está muy extendida, aún dentro de la misma población sorda (Woodward 1980).

a. Esta actitud se puede deber al hecho de que es más fácil para un sordo que para un oyente cuando viaja al extranjero, hacerse entender mediante la pantomima sin recurrir necesariamente a la lengua de señas o a la lengua oral. Y aunque es menos rápida y se reduce la eficiencia en la comunicación, a un observador le puede dar la impresión de que los sordos tienen una lengua común de señas.

b. Por otra parte, aunque hay características universales entre las lenguas, cada una tiene un desarrollo muy particular, basta con revisar diccionarios de distintas lenguas de señas para darse cuenta de que no todos los grupos de sordos denotan las mismas cosas con las mismas señas (Battison 1978). Tal es el caso de la señas para VENADO, MUJER, POR-FAVOR ${ }^{5}$, las cuales se articulan de forma distinta en la lengua de señas de Nicaragua y en el LESCO.
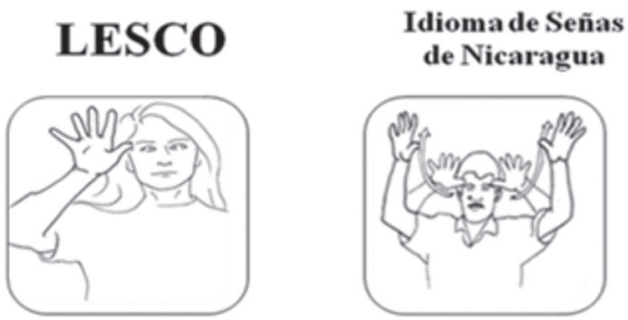

VENADO
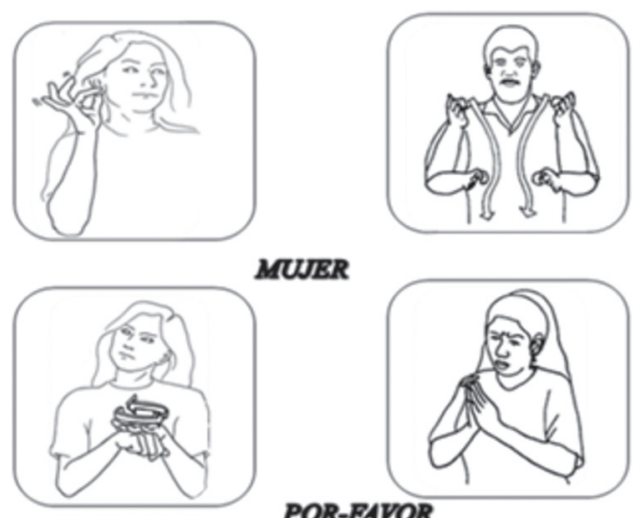

MUUER

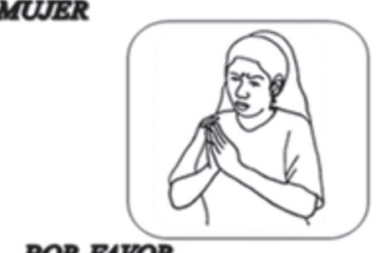

POR-FAVOR

Figura 5. Formación de las señas en LESCO y lengua de señas de Nicaragua. 
Por lo tanto, las características que aproximen o distancien a dos lenguas dependerá del grado de parentesco existente entre estas; es decir, del contacto lingüístico dado a través del tiempo. Por ejemplo, véase la relación existente entre la lengua norteamericana de señas y la lengua francesa de señas, la cual surge en 1815, cuando Thomas Hopkins Gallaudet, después de estudiar todo un año en París con Sicard, aprendió las técnicas para educar al sordo y junto con un estudiante sordo francés, Laurent Clerc, regresa a Hartford Connecticut para establecer el método de instrucción l'Epée.

La evidencia existente permite pensar que por lo menos algunos de los sordos de esa época tuvieron conocimiento en Norteamérica de otras lenguas antes de que Clerc empezara a enseñar las señas francesas ${ }^{6}$ y es muy probable que los sordos terminaran combinando algunas de las señas de su lengua con las señas francesas y de esta mezcla surgiera la antigua lengua norteamericana de señas.

\section{Reseña histórica de la lengua de señas}

Sobre el origen del LESCO es poco lo que se conoce antes de 1939. Sin embargo, Woodward (2010) sugiere que esta lengua probablemente se originó por el contacto entre la lengua de señas española y las señas autóctonas de Costa Rica.

Esta situación se produce en una época anterior al establecimiento de la educación del sordo en nuestro país, cuando algunos jóvenes viajan a España para educarse. Allí, en su interacción cotidiana con otros sordos, tienen contacto con la lengua de señas española y a su regreso traen consigo señas que se mezclan con las señas autóctonas de San José.

Posteriormente, la fundación de la Escuela de Enseñanza Especial Fernando Centeno Güell en 1940, tiene una importancia fundamental en el desarrollo de esta lengua. Aunque se aplica el método oralista para enseñarle al sordo a articular los sonidos de la mejor manera posible y se deja de lado la enseñanza o el uso de las señas en clase, este centro educativo se convierte en el primer lugar de encuentro importante para los sordos jóvenes que estudian en San José, quienes comparten sus señas aunque sea en los recreos.

Con el pasar del tiempo, los sordos establecen otros lugares para interactuar que permiten el nacimiento de nuevas señas y su aceptación dentro de la comunidad sorda costarricense. A partir de los años sesentas, el tramo de Lolo Mora, en el Mercado Central sirvió como punto de convergencia para los sordos jóvenes de San José, quienes se reunían allí con Roberto Castro, nieto de Lolo Mora, y se comunicaban entre ellos mediante el uso de las señas. Por lo tanto, en las inmediaciones de este tramo josefino, surgen muchas de las señas de esta lengua, las cuales poco a poco se van a ir estandarizando.

En 1974, se formaliza la fundación de la Asociación Deportiva Silenciosa de Costa Rica alrededor de María Infante, Rafael Eduardo Valverde y Fernando Zúñiga. Esta asociación, ubicada en el Mercado Central, funciona a partir de aquel momento como un lugar más de reunión para los miembros de la comunidad sorda de San José.

Posteriormente, tanto en San José como en otras provincias, la comunidad sorda joven ha buscado puntos de encuentro para interactuar en LESCO. En San José, por ejemplo, muchos sordos se reúnen en las inmediaciones de la McDonald's en la Plaza de la Cultura en San José desde la década de los ochentas hasta el día de hoy, mientras que en las cabeceras de provincia, suelen reunirse en los parques un día determinado de la semana. 


\subsection{Primera recopilación léxica}

De acuerdo con la doctora Soledad Chavarría, el primer esfuerzo por registrar el vocabulario básico de la lengua costarricense de señas se da en el año de 1974, cuando el Dr. Gilbert Delgado, profesor de la Universidad de Gallaudet, llega a nuestro país con el propósito de estudiar el estado del adulto sordo. En este viaje, lo acompañan dos lingüistas de la misma universidad que recopilan el léxico básico de la lengua de señas costarricense, el cual es filmado en película de $8 \mathrm{~mm}$. La visita del Dr. Delgado trajo consigo el establecimiento de nexos entre Costa Rica y la Universidad de Gallaudet ${ }^{7}$.

En 1976, Ermida Bravo Stahl, profesora de Educación Especial, recibe una beca del Dr. Delgado, para diseñar junto a él un proyecto que consigne el léxico de la lengua de señas de Costa Rica, el cual sería financiado con fondos de la OEA. Para realizarlo, Mima Bravo se traslada a la Universidad de Gallaudet en compañía de los costarricenses Rafael Valverde, señante nativo del LESCO y Gloria Campos Centeno, quien ese mismo año acababa de regresar de Lexington, Nueva York. En un esfuerzo conjunto, logran registrar 500 señas mediante fotografías, con las que publican en 1977 el diccionario Hacia una nueva forma de comunicación con el sordo.

Al regresar a Costa Rica, traen consigo la experiencia obtenida por la realización de este proyecto y muchas señas que tomaron como préstamos lingüísticos, debido al impacto que les produjo el contacto con la lengua norteamericana de señas. Por el liderazgo que tenía Rafael Valverde entre los miembros jóvenes de la comunidad sorda, logra la aceptación de las señas extranjeras y el paulatino desuso de las señas autóctonas que se han sustituido.

El influjo de la lengua de señas norteamericana sobre la lengua de señas costarricense da inicio a una variación en la lengua que produce un gran contraste entre las señas de las generaciones que en aquel momento tienen más de 30 años y las generaciones de menos de 30 años. Los primeros conservan las señas tradicionales y se siguen reuniendo en las inmediaciones del Mercado Central, mientras que los últimos, educados con las nuevas señas, se adaptan y crecen con ellas.

Muchos sordos jóvenes en la década de los noventas, de acuerdo con las observaciones registradas, establecían la diferencia entre señas viejas y señas nuevas; asimismo, consideraban que estas últimas tenían más prestigio social entre su grupo.

\subsection{Investigaciones lingüísticas relacionadas con el LESCO}

En la década de los noventa, surgen los primeros estudios lingüísticos del LESCO. Woodward (1992) publica dos artículos relacionados con el origen y las relaciones de parentesco entre esta lengua y las que le dieron origen. Luego tenemos mi tesis de maestría, Retana (1993), en esta obra, se realiza una descripción del aspecto verbal en el LESCO; además, se incluye una breve descripción de la estructura básica de la seña y se ilustran algunos procesos morfológicos de la lengua. Sin embargo, esta documentación linguiística sólo puede consultarse en algunas bibliotecas de la Universidad de Costa, ya que no se ha digitalizado, lo cual invisibiliza la información para quien desee revisarla en internet, aquí o en cualquier parte del mundo.

Después de estas aproximaciones iniciales a la lengua, pasan más de 10 años antes de que aparezca otro estudio. Sánchez, precisamente, en el 2005, hace referencia a la lengua de señas costarricense al reflexionar sobre lo que debería ser el modelo de la educación bilingüe 
para la población sorda. Más tarde, en el 2009, al describir los distintos grupos de lenguas minoritarias de Costa Rica, comenta algunos aspectos sociolingüísticos del LESCO.

De las investigaciones realizadas en estos veinte años, es importante retomar el estudio lexicográfico realizado por el lingüista norteamericano James Woodward (1992) quien en una de sus visitas a Costa Rica en 1990 observa semejanzas entre la realización de varias formas léxicas de la lengua norteamericana de señas y la lengua de señas que utilizan los jóvenes sordos.

De acuerdo con su perspectiva, el investigador establece la existencia de dos variedades lingüísticas de la lengua de señas costarricense y las denomina en inglés como "Original LESCO" (antigua lengua de señas de Costa Rica) y "NLESCO” (nueva o moderna lengua de señas de Costa Rica), siendo esta última la que la mayor parte de la población sorda llama

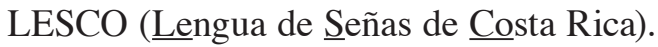

Ambas formas son utilizadas por señantes del área metropolitana, principalmente. Pero, mientras que la antigua lengua de señas costarricense es usada por sordos que en la década de los noventas tenían más de treinta años, la lengua de señas actual la utilizan los menores de treinta años. Estos hallazgos lo motivan a estudiar las relaciones históricas de la actual lengua de señas costarricense y las lenguas de las cuales procede: la lengua de señas norteamericana y la antigua lengua de señas costarricense.

Con este propósito, selecciona como informantes a dos señantes nativos de San José, madre e hijo, ambos son sordos por herencia. La madre de unos 44 años de edad maneja las señas tradicionales, mientras que su hijo, de unos 25 años, adquiere las señas maternas y al crecer y vivir en San José, aprende y usa las señas del NLESCO. Utiliza una lista de vocabulario especial para la investigación de lenguas de señas que él mismo derivó de la lista de 200 palabras de Swadesh con la cual le pide a cada informante la seña respectiva.

Su estudio revela que la nueva lengua de señas de Costa Rica (NLESCO) es una lengua distinta de sus progenitoras y mantiene una relación histórica más cercana con la lengua de señas norteamericana (LSN) que con la antigua lengua de señas de Costa Rica (OLESCO). Por otra parte, señala que vocabulario básico proviene de distintas fuentes: una cuarta parte de este proviene tanto del OLESCO como de la LSN.

De acuerdo con Woodward, estas lenguas progenitoras son distintas y entre ellas hay una relación histórica mínima. La lengua norteamericana de señas ha tenido, en su desarrollo, influencia de la lengua de señas francesa, la cual está bien documentada. Y, probablemente, hubo una influencia indirecta de las lenguas de señas de España y Francia, debido a que antes de que se estableciera la educación del sordo en 1940, algunos jóvenes costarricenses estudiaron en España, donde probablemente tomaron alguna lengua de señas española y la trajeron a Costa Rica donde se mezcló con las señas indígenas del segmento hispánico de San José.

Del vocabulario básico del NLESCO, por otro lado, solo un $16.3 \%$ de este, una sexta parte, procede del OLESCO, mientras que más del doble de ese porcentaje, un $37.8 \%$, se tomó en calidad de préstamo de la lengua de señas norteamericana en menos de 30 años.

Llama la atención del autor que un 20.4\% del vocabulario básico del NLESCO no parece venir del OLESCO ni de la lengua norteamericana de señas. El autor presupone que podría venir de otras variedades de la lengua costarricense de señas que no han sido investigadas o podría ser que estas señas se crearan a partir de un proceso de criollización de la lengua norteamericana de señas con varias formas de OLESCO.

En un artículo posterior (2010), Woodward menciona que el NLESCO al igual que otras lenguas de señas modernas se ha formado por la mezcla de una o más lenguas de señas originales y una extranjera introducida en el sistema educativo. 


\section{Conclusiones}

En estos momentos, no solo es importante retomar el estudio de la lengua de señas sino también difundir más allá del ámbito académico los resultados que generen las investigaciones, con el fin de propiciar un cambio de actitud en la mayoría de los individuos hacia la comunidad sorda y su lengua.

\section{Notas}

1. Recuerden que en 1993 apenas iniciaba la conexión de internet en el ámbito académico en nuestro país.

2. Las lenguas de señas utilizan las manos, la expresión facial y los movimientos corporales para la producción de las señas y su recepción se realiza por medio de la vista.

3. Las ilustraciones del LESCO le pertenecen a la autora y se diseñaron con base en fotografías tomadas a una de las informantes en 1992.

4. William Stokoe, lingüista norteamericano y precursor del estudio de las lenguas de señas, propone un modelo de análisis para describir las lenguas visogestuales y la estructura de los signos lingüísticos, el cual ha servido como referencia a distintos marcos teóricos que se han desarrollado en décadas posteriores para estudiar la estructura de estas lenguas.

5. En la figura 5, se utilizaron imágenes del Diccionario del Idioma de Señas de Nicaragua de ANSNIC (1997).

6. En relación con este tema, Carol Padden (Cfr.2010) comenta que en la isla de Martha‘s Vineyard existió una comunidad sorda que tenían su propia lengua de señas, mucho antes de la fundación de una escuela para sordos, lo cual ejemplifica el hecho de que las lenguas de señas como cualquier lengua natural se crean en la colectividad y se transmiten de generación en generación.

7. Esta institución educa solo a sordos desde el jardín de infantes hasta posgrados -y sólo en los posgrados se aceptan oyentes.

\section{Bibliografía}

Asociación Nacional de Sordos de Nicaragua (ANSNIC). 1997. Diccionario del Idioma de Señas de Nicaragua. Managua: Copy Fast S.A.

Battison, Robin. 1978. Lexical Borrowing in American Sign Language. Maryland: Linstok Press.

Gaurav Mathur y Donna Jo Napoli (Eds.). 2010. Deaf around the world: The impact of Language. Estados Unidos: Oxford University Press.

Klima, Edward y Ursula Bellugi. 1979. The signs of language. Cambridge: Harvard University Press. 
Padden, Carol. 2010. “Sign language geography”. En: Gaurav y Napoli (Eds.), 19-37.

Pietrosemoli, Lourdes. 1988. Señas y palabras. Mérida: Consejo de Publicaciones de la Universidad de Los Andes.

Retana Castro, Priscilla. 1993. Descripción del aspecto verbal en la lengua costarricense de señas. Tesis de Maestría: Universidad de Costa Rica.

Sánchez Avendaño, Carlos. 2005. "El español y el LESCO en el marco de la enseñanza de una segunda lengua para las personas sordas en Costa Rica”. Revista Educación. 29 (2): 217-232.

2009. "Situación sociolingüística de las lenguas minoritarias de Costa Rica y censos nacionales de población 1927-2000: vitalidad, desplazamiento y autoafiliación etnolingüística. Revista de Filología y Lingüística 35 (2): 233-273.

Woodward, James. 1982. "Beliefs About and Attitudes Toward Deaf People and Sign Language on Providence Island". How You Gonna Get to Heaven if You Can't Talk to Jesus? Silver Spring, MD: T.J. Publishers.

1992. "Historical Bases of New Costa Rican Sign Language”. Revista de Filología y Lingüística. 17 (1):127-132.

2010. "Some Observations on Research Methodology in Lexicostatistical Studies of Sign Language” En: Gaurav y Napoli (Eds.), 38-53. 